\title{
FLEXIBLE ALGEBRAS OF DEGREE TWO
}

\author{
BY \\ JOSEPH H. MAYNE( $\left.{ }^{1}\right)$
}

\begin{abstract}
All known examples of simple flexible power-associative algebras of degree two are either commutative or noncommutative Jordan. In this paper we construct an algebra which is partially stable but not commutative and not a noncommutative Jordan algebra. We then investigate the multiplicative structure of those algebras which are partially stable over an algebraically closed field of characteristic $p \neq 2,3,5$. The results obtained are then used to develop conditions under which such algebras must be commutative.
\end{abstract}

I. Introduction. An algebra $A$ over a field $F$ is flexible if it satisfies the identity

$$
(x, y, x)=0 \text { for all } x, y \text { in } A \text { where }(a, b, c)=(a b) c-a(b c) .
$$

If the characteristic of $F$ is not two, then this identity is equivalent to

$$
(x, y, z)+(z, y, x)=0 \text { for all } x, y, z \text { in } A \text {, }
$$

the linearized form of the flexible law. If any element in $A$ generates an associative subalgebra, then $A$ is power-associative and $A$ is strictly powerassociative if the algebra $A_{K}=K \otimes_{F} A$ is power-associative for every extension field $K$ of $F$. We shall assume that all algebras are finite dimensional over the base field.

If $A$ is power-associative and contains an idempotent $u$, then $A$ has a Peirce decomposition

$$
A=A_{u}(1)+A_{u}(1 / 2)+A_{u}(0) \quad \text { (vector space direct sum) }
$$

where $A_{u}(s), s=0,1 / 2,1$, is a subspace of $A$ such that $x$ is in $A_{u}(s)$ if and only if $x u+u x=2 s x$. Moreover, if $A$ is flexible, the multiplicative relations

$$
A_{u}(0) A_{u}(1)=A_{u}(1) A_{u}(0)=0
$$

Prese nted to the Society, January 21,1971 ; received by the editors June 10,1971 .

AMS 1970 subject classifications. Primary 17A05, 17A20; Secondary 17A15, 17 A10.

Key words and phrases. Flexible algebras, power-associative algebras, degree two, stable, noncommutative Jordan algebras.

(1) The results of this paper are a part of the author's doctoral dissertation at the Illinois Institute of Technology, written under the direction of Professor Louis A. Kokoris. This research was supported by a National Aeronautics and Space Administration Traineeship. 
and

$$
\begin{array}{ll}
A_{u}(s) A_{u}(1 / 2) \subseteq A_{u}(1 / 2)+A_{u}(1-s) & (s=0,1), \\
A_{u}(1 / 2) A_{u}(s) \subseteq A_{u}(1 / 2)+A_{u}(1-s) & (s=0,1), \\
A_{u}(s) A_{u}(s) \subseteq A_{u}(s) & (s=0,1),
\end{array}
$$

are satisfied [1].

A flexible algebra $A$ is $u$-stable if

$$
\begin{array}{ll}
A_{u}(s) A_{u}(1 / 2) \subseteq A_{u}(1 / 2) & (s=0,1), \\
A_{u}(1 / 2) A_{u}(s) \subseteq A_{u}(1 / 2) & (s=0,1) .
\end{array}
$$

If $A$ contains an idempotent $u$ such that $A$ is $u$-stable, $A$ is said to be partially stable and if $A$ is $u$-stable for every idempotent $u$ in $A$, then $A$ is termed a stable algebra.

An idempotent $u$ is said to be primitive if in the Peirce decomposition $A_{u}(1)$ contains no other idempotent besides $u$. Now every simple flexible strictly power-associative algebra with characteristic not 2,3 has a unity element 1 [13]. Let $t$ be the maximal number of pairwise orthogonal primitive idempotents $u_{1}, \cdots, u_{t}$ in any scalar extension $A_{K}$ of $A$ such that $1=u_{1}+\cdots+u_{t}$, then $t$ is called the degree of $A$.

A simple flexible strictly power-associative algebra over a field $F$ of characteristic not 2 or 3 is one of the following [13]:

(i) a commutative Jordan algebra,

(ii) a quasi-associative algebra,

(iii) an algebra of degree one,

(iv) an algebra of degree two.

Kleinfeld and Kokoris have shown that the degree one algebras are fields if the characteristic of $F$ is 0 [8], and Oehmke [15] has recently proved that if $F$ is an algebraically closed field of characteristic not two, then a degree one algebra is either a field or a nodal noncommutative algebra of the type described by Kokoris in [11]. Oehmke [14] and Kokoris [12] have studied the degree two algebras and it is known that if $A$ is nilstable and $F$ is an algebraically closed field of characteristic not 2,3 , or 5 , then $A$ is a $J$-simple algebra. In this paper we shall show that this result cannot be extended to arbitrary degree two algebras.

Let $\mathfrak{f}$ be the class of partially stable power-associative flexible algebras of degree two which are simple over an algebraically closed field of characteristic not 2,3 , or 5 . We shall show that algebras in $\mathcal{F}$ need not be commutative 
and in addition need not be noncommutative Jordan algebras. This is accomplished by constructing an example of such an algebra. Finally we develop conditions under which an algebra in the class $\mathfrak{F}$ must be commutative.

II. New algebras of degree two. In 1952 Kokoris [9] constructed the first known example of a simple commutative power-associative algebra which was not a Jordan algebra. Albert [3], [4], [5] generalized this work and characterized all degree two algebras which are partially stable over an algebraically closed field. We begin by constructing an algebra in this class.

Let $F$ be an algebraically closed field of characteristic $p \neq 2,3,5$ and let $B=F_{p}[1, x]$ be the commutative associative polynomial algebra with identity on one generator subject to the relation $x^{p}=0$. Suppose $z B, y_{0} B, y_{1} B, y_{2} B$ and $y_{3} B$ are isomorphic copies of $B$ and then form the vector space direct sum $A=B+z B+y_{0} B+y_{1} B+y_{2} B+y_{3} B$. Define

$$
\begin{gathered}
y_{0}^{2}=1, \quad y_{0} y_{1}=0, \quad y_{1} y_{2}=1, \\
y_{1}^{2}=0, \quad y_{0} y_{2}=0, \quad y_{1} y_{3}=0, \\
y_{2}^{2}=0, \quad y_{0} y_{3}=0, \quad y_{2} y_{3}=0, \\
y_{3}^{2}=0 \quad \text { and } y_{i} y_{j}=y_{j} y_{i} \quad \text { for } i, j=0,1,2,3 .
\end{gathered}
$$

Now let $\supseteqq=\left\{D_{i j}: i, j=0,1,2,3\right\}$ be a set derivations on $B$ given by

$$
\begin{gathered}
x D_{01}=0, \quad x D_{02}=0, \quad x D_{03}=0, \\
x D_{12}=0, \quad x D_{13}=1, \quad x D_{23}=0, \\
D_{i j}=-D_{j i} \quad(i, j=0,1,2,3) .
\end{gathered}
$$

Then define multiplication on $A$ by assuming for $i, j=0,1,2,3$ and $a, b$ in $B$ that

(3) $\left(y_{i} a\right)\left(y_{j} b\right)=\left(y_{i} y_{j}\right)(a b)+\left(a D_{i j}\right) b-a\left(b D_{i j}\right)$,

(4) $\left(y_{i} a\right) b=b\left(y_{i} a\right)=y_{i}(a b)$,

(5) $(z a)\left(y_{i} b\right)=\left(y_{i} b\right)(z a)=0$,

(6) $B+z B$ is a commutative and associative subalgebra of $A$,

(7) $(z a)(z b)=a b$.

It is shown in [4] that such an algebra is commutative, power-associative and of degree two over $F . A$ is $u$-stable where $u=1 / 2(z+1)$ and if $A=$ $A_{1}+A_{1 / 2}+A_{0}$ is the Peirce decomposition of $A$ with respect to $u$, then $A_{1 / 2}=y_{0} B+y_{1} B+y_{2} B+y_{3} B$ and $A_{1}+A_{0}=B+z B$. 
Lemma 1. The algebra $A$ is simple.

Proof. It is known [4, Theorem 3] that $A$ is simple if and only if $B$ is I-simple and there exists no element $g$ in $A_{1 / 2}$ such that $g d=0$ for all $d$ in $A_{1 / 2}$.

Suppose $y=y_{0} f+y_{1} g+y_{2} b+y_{3} k$, where $f, g, b, k$ are in $B$, is an element in $A_{1 / 2}$ such that $y d=0$ for all $d$ in $A_{1 / 2}$. In particular $y y_{0}=y_{0}^{2} f=f=0$ and so $y=y_{1} g+y_{2} b+y_{3} k$. Then $y y_{2}=\left(y_{1} y_{2}\right) g=g=0$, thus $y=y_{2} b+y_{3} k$.

Now $y_{1} y=y_{1}\left(y_{2} b+y_{3} k\right)=b-k^{\prime}=0$ where $k^{\prime}$ is just the formal derivative of $k$ with respect to $x$. Hence $b=k^{\prime}$ and so $y=y_{2} k^{\prime}+y_{3} k$. But $\left(y_{1} x\right) y=$ $\left(y_{1} x\right)\left(y_{2} k^{\prime}+y_{3} k\right)=x k^{\prime}+k-x k^{\prime}=k=0$ and thus $b=0$ and $y=0$. Since $B$ has no proper ideals invariant under $D_{13}, A$ is a simple algebra.

Now denote the multiplication described above by $x \cdot y$ and let $[x, y]$ be the image of a bilinear function from $A \times A$ into $A$ with the following properties:

(8) $[x, x]=0$ for all $x$ in $A$,

(9) $[a, b]=0$ for all $a, b$ in $A_{1}+A_{0}$,

(10) $\left[b, a_{1 / 2}\right]=0$ for all $b$ in $B$ and $a_{1 / 2}$ in $A_{1 / 2}$,

(11) $\left[y_{i} \cdot a, z \cdot b\right]=\left[y_{i}, z\right] \cdot(a \cdot b)$ for $a, b$ in $B(i=0,1,2,3)$,

(12) $\left[y_{i} \cdot a, y_{j} \cdot b\right]=\left[y_{i}, y_{j}\right] \cdot(a \cdot b)$ for $a, b$ in $B(i, j=0,1,2,3)$,

$$
\begin{array}{lll}
{\left[y_{0}, u\right]=y_{2},} & {\left[y_{0}, y_{1}\right]=-2 z,} & {\left[y_{1}, y_{2}\right]=0,} \\
{\left[y_{1}, u\right]=-y_{0},} & {\left[y_{0}, y_{2}\right]=0,} & {\left[y_{1}, y_{3}\right]=0,} \\
{\left[y_{2}, u\right]=0,} & {\left[y_{0}, y_{3}\right]=0,} & {\left[y_{2}, y_{3}\right]=0,} \\
{\left[y_{3}, u\right]=0 .} & &
\end{array}
$$

Let $x y=x \cdot y+1 / 2[x, y]$ for all $x, y$ in $A$ and from now on consider $A$ as an algebra with product $x y$. Then the attached algebra $A^{+}$with multiplication $x \cdot y=1 / 2(x y+y x)$ is just the commutative algebra defined prior to Lemma 1 and $[x, y]=x y-y x$ is the commutator product on $A$. Since $A^{+}$is simple and $u$-stable, $A$ must also be simple and $u$-stable. We now show that $A$ is a flexible algebra.

Lemma 2. The algebra $A$ is flexible.

Proof. Now $A$ is a flexible algebra if and only if the linear mapping $D_{x}$ defined by $y D_{x}=[y, x]$ is a derivation on $A^{+}$. This is equivalent to the identity

$$
[w \cdot x, y]-w \cdot[x, y]-x \cdot[w, y]=0 \text {. }
$$

Hence it is sufficient to show that (13) is satisfied when all possible combinations of elements from the subspaces $B, z B$, and $A_{1 / 2}$ are substituted for $w, x$, and $y$.

Now if $w, x$, and $y$ are all in $B+z B$, then (13) is satisfied since $B+z B=$ 
$A_{1}+A_{0}$ is a commutative associative subalgebra of $A$. We also know from (3) that $\left(y_{i} a\right) \cdot\left(y_{j} b\right)$ is in $B$ for $a, b$ in $B$ and $i, j=0,1,2,3$. From now on let us assume that multiplications in $A$ are to be performed before multiplications in $A^{+}$unless otherwise specified so that $w z \cdot x y=(w z) \cdot(x y)$.

Suppose $w=y_{i} a, x=y_{j} b$, and $y=y_{k} c$ where $a, b$, and $c$ are in $B$. Then $[w \cdot x, y]=\left[y_{i} a \cdot y_{j} b, y_{k} c\right]=0$ because of (10). Also $w \cdot[x, y]=y_{i} a \cdot\left[y_{j} b, y_{k} c\right]$ $=0$ and $x \cdot[w, y]=y_{j} b \cdot\left[y_{i} a, y_{k} c\right]=0$ by (5), (12), and the way the multiplications $\left[y_{i}, y_{j}\right](i, j=0,1,2,3)$ are defined. Thus in this case (13) holds and so $A_{1 / 2}$ is flexible.

Consider (13) for elements in $B$ and $A_{1 / 2}$. If two of the three variables $w, x$, and $y$ are in $B$, then each commutator in (13) has at least one entry from $B$ and hence vanishes since $B$ commutes with everything in $A$ by (9) and (10).

Now suppose $w=y_{i} a, x=y_{j} b, y=c$ where $a, b$, and $c$ are in $B$. Then (13) is satisfied since $c$ commutes with everything in $A$. If $w=y_{i} a, x=b$, and $y=y_{k} c$, then the left side of (13) is

$\left[y_{i} a \cdot b, y_{k} c\right]-y_{i} a \cdot\left[b, y_{k} c\right]-b \cdot\left[y_{i} a, y_{k} c\right]=\left[y_{i}, y_{k}\right](a b c)-\left[y_{i}, y_{k}\right](a b c)=0$.

Since (13) is symmetric in $x$ and $w$, this exhausts the possibilities for elements from $B$ and $A_{1 / 2}$.

Now suppose one element comes from each of the subspaces $B, z B$, and $A_{1 / 2}$. Because of the symmetry of (13) there are only three possibilities:

(i) $w=y_{i} a, x=b, y=z c$;

(ii) $w=z a, x=b, y=y_{k} c$;

(iii) $w=z a, x=y_{j} b, y=c$; where $a, b$, and $c$ are in $B$.

For case (i): $\left[y_{i} a \cdot b, z c\right]-y_{i} a \cdot[b, z c]-b \cdot\left[y_{i} a, z c\right]=\left[y_{i}, z\right](a b c)-$ $\left[y_{i}, z\right](a b c)=0$ by (11). Case (ii) is similat and in case (iii), (13) is satisfied since $c$ commutes with everything in $A$.

All that remains to be checked is that (13) is satisfied by combinations of elements from $z B$ and $A_{1 / 2}$. Because of the symmetry of (13) there are only four possible combinations:

(i) $w=y_{i} a, x=z b, y=z c$;

(ii) $w=z a, x=z b, y=y_{k} c$;

(iii) $w=y_{i} a, x=z b, y=y_{k} c$;

(iv) $w=y_{i} a, x=y_{j} b, y=z c$.

For case (i): $\left[y_{i} a \cdot z b, z c\right]-y_{i} a \cdot[z b, z c]-z b \cdot\left[y_{i} a, z c\right]=-z b \cdot\left[y_{i}, z\right](a c)=0$ by (9), (11), the definition of $\left[y_{i}, z\right]$, and multiplication in $A^{+}$. Similarly cases (ii)-(iv) satisfy (13). Hence $A$ is a flexible algebra.

Before proving that $A$ is power-associative, we prove a lemma relating power-associativity in $A^{+}$and $A$. Note that in a flexible algebra $A, x^{2} x=x x^{2}$ so $x^{3}$ is well defined. 
Lemma 3. If $C$ is a flexible algebra over a field of characteristic not 2, 3, or 5 and $C^{+}$is power-associative, then $C$ is power associative if and only if $\left[x^{3}, x\right]=0$.

Proof. If $C$ is power-associative then $\left[x^{3}, x\right]=0$ by definition. Now suppose $\left[x^{3}, x\right]=0$, then $x^{3} x=x x^{3}$ or $2 x^{3} x=x x^{3}+x^{3} x=2 x \cdot x^{3}$. Now if $C^{+}$ is power-associative, $x \cdot x^{3}=\left(x^{2} \cdot x\right) \cdot x=x^{2} \cdot x^{2}$. But $x^{2} \cdot x^{2}=x^{2} x^{2}$ and hence $x^{3} x=\left(x^{2} x\right) x=x^{2} x^{2}$. Therefore $C$ is power-associative [1].

Lemma 4. The algebra $A$ is power-associative.

Proof. Let $x=y_{0} a+y_{1} b+y_{2} c+y_{3} d+z f+g$ be an arbitrary element in $A$ where $a, b, c, d, f$, and $g$ are in $B$. Then

$$
\begin{aligned}
x^{2}= & \left(y_{0} a\right)^{2}+\left(y_{1} b\right)^{2}+\left(y_{2} c\right)^{2}+\left(y_{3} d\right)^{2}+(z f)^{2}+g^{2} \\
& +2\left(y_{0} a\right) \cdot\left(y_{1} b\right)+2\left(y_{0} a\right) \cdot\left(y_{2} c\right)+2\left(y_{0} a\right) \cdot\left(y_{3} d\right) \\
& +2\left(y_{0} a\right) \cdot(z f)+2\left(y_{0} a\right) \cdot g+2\left(y_{1} b\right) \cdot\left(y_{2} c\right) \\
& +2\left(y_{1} b\right) \cdot\left(y_{3} d\right)+2\left(y_{1} b\right) \cdot(z f)+2\left(y_{1} b\right) \cdot g \\
& +2\left(y_{2} c\right) \cdot\left(y_{3} d\right)+2\left(y_{2} c\right) \cdot(z f)+2\left(y_{2} c\right) \cdot g \\
& +2\left(y_{3} d\right) \cdot(z f)+2\left(y_{3} d\right) \cdot g+2(z f) \cdot g \\
= & 2 y_{0}(a g)+2 y_{1}(b g)+2 y_{2}(c g)+2 y_{3}(d g) \\
& +2 z(f g)+a^{2}+f^{2}+g^{2}+2 b c+2\left(b^{\prime} d-d^{\prime} b\right)
\end{aligned}
$$

where $b^{\prime}$ is again the formal derivative of $b$ with respect to $x$.

Let $b=a^{2}+f^{2}+g^{2}+2 b c+2\left(b^{\prime} d-d^{\prime} b\right)$, then

$$
\begin{aligned}
x^{2} x= & {\left[2 y_{0}(a g)+2 y_{1}(b g)+2 y_{2}(c g)+2 y_{3}(d g)+2 z(f g)+b\right] } \\
& \cdot\left(y_{0} a+y_{1} b+y_{2} c+y_{3} d+a f+g\right) \\
= & 2 a^{2} g-2 z(a g b)+2 y_{2}(a g f)+2 y_{0}\left(a g^{2}\right)+2 z(b g a)+2 b g c \\
& +2\left[(b g)^{\prime} d-b g d^{\prime}\right]-2 y_{0}(b g f)+2 y_{1}\left(b g^{2}\right)+2 b g c+2 y_{2}\left(c g^{2}\right) \\
& +2\left[b^{\prime} d g-(d g)^{\prime} b\right]+2 y_{3}\left(d g^{2}\right)-2 y_{2}(f g a)+2 y_{0}(f g b) \\
& +2 f^{2} g+2 z f g+y_{0}(a b)+y_{1}(b b)+y_{2}(c b)+y_{3}(d b)+z(f b)+g b \\
= & y_{0}\left(2 a g^{2}+a b\right)+y_{1}\left(2 b g^{2}+b b\right)+y_{2}\left(2 c g^{2}+c b\right)+y_{3}\left(2 d g^{2}+d b\right) \\
& +z\left(2 f g^{2}+f b\right)+4\left(b^{\prime} d g-d^{\prime} g b\right)+4 b g c+2 a^{2} g+2 f^{2} g+g b .
\end{aligned}
$$

Let

$$
\begin{array}{ll}
a_{1}=2 a g^{2}+a b, & b_{1}=2 b g^{2}+b b, \quad c_{1}=2 c g^{2}+c b, \quad d_{1}=2 d g^{2}+d b, \\
f_{1}=2 f g^{2}+f b, & g_{1}=4\left(b^{\prime} d g-d^{\prime} g b\right)+4 b g c+2 a^{2} g+2 f^{2} g+g b,
\end{array}
$$


then

$$
x^{2} x=x^{3}=y_{0} a_{1}+y_{1} b_{1}+y_{2} c_{1}+y_{3} d_{1}+z f_{1}+g_{1}
$$

and

$$
\begin{aligned}
{\left[x^{3}, x\right] } & =\left[y_{0} a_{1}+y_{1} b_{1}+y_{2} c_{1}+y_{3} d_{1}+z f_{1}+g_{1}, y_{0} a+y_{1} b+y_{2} c+y_{3} d+z f+g\right] \\
& =-2 z a_{1} b+2 y_{2} a_{1} f+2 z b_{1} a-2 y_{0} b_{1} f-2 y_{2} f_{1} a+2 y_{0} f_{1} b \\
& =2 y_{0}\left(f_{1} b-b_{1} f\right)+2 y_{2}\left(a_{1} f-f_{1} a\right)+2 z\left(b_{1} a-a_{1} b\right) .
\end{aligned}
$$

Now

$$
\begin{aligned}
& f_{1} b-b_{1} f=2 f g^{2} b+b f b-2 b g^{2} f-b b f=0, \\
& a_{1} f-f_{1} a=2 a g^{2} f+a b f-2 f g^{2} a-f b a=0,
\end{aligned}
$$

and

$$
b_{1} a-a_{1} b=2 b g^{2} a+b b a-2 a g^{2} b-a b b=0,
$$

hence $\left[x^{3}, x\right]=0$. Since $A^{+}$is power-associative, by Lemma 3 , we have that $A$ is power-associative.

Theorem 1. A is a simple power-associative flexible algebra of degree two which is not commutative.

Proof. We have already shown that $A$ is simple, flexible, and powerassociative. $A$ is of degree two since $A^{+}$is a degree two algebra and $A$ is flexible. $A$ is not commutative since $y_{0} u=u y_{0}+y_{2}$ and $y_{2} \neq 0$.

Although $A$ is partially stable it is not stable and hence not a noncommutative Jordan algebra. We show this in Theorem 2.

Theorem 2. $A$ is not a noncommutative Jordan algebra.

Proof. It is sufficient to show that $A^{+}$is not stable and hence not a Jordan algebra. Denote multiplication in $A^{+}$by $x y$. Let $w=y_{1} x+1 / 2 y_{3}$, then $w^{2}=$ $\left(y_{1} x+1 / 2 y_{3}\right)^{2}=\left(y_{1} x\right) y_{3}=1$. If $e=1 / 2(1+w)$, then $e^{2}=1 / 4\left(1+2 w+w^{2}\right)=$ $1 / 2(1+w)=e$, and so $e$ is an idempotent in $A$. Now $y_{1} e=y_{1}\left(1 / 2+1 / 2 y_{1} x+1 / 4 y_{3}\right)=$ $1 / 2 y_{1}$ and so $y_{1}$ is in $A_{e}(1 / 2)$.

Also $(e x) e=\left(1 / 2 x+1 / 2 y_{1} x^{2}+1 / 4 y_{3} x\right)\left(1 / 2+1 / 2 y_{1} x+1 / 4 y_{3}\right)=1 / 2 x+1 / 2 y_{1} x^{2}+1 / 4 y_{3} x=$ $e x$ and thus $e x$ is in $A_{e}(1)$. But if we consider

$$
\begin{aligned}
{\left[y_{1}(e x)\right] e } & =\left[y_{1}\left(1 / 2 x+1 / 2 y_{1} x^{2}+1 / 4 y_{3} x\right)\right]\left(1 / 2+1 / 2 y_{1} x+1 / 4 y_{3}\right) \\
& =1 / 4\left(1 / 2 y_{1} x-1 / 4 y_{3}\right) \neq 1 / 2\left[y_{1}(e x)\right],
\end{aligned}
$$

then we see that $y_{1}(e x)$ is not in $A_{e}(1 / 2)$ and hence $A^{+}$is not $e$-stable.

III. Algebras in the class $\mathfrak{F}$. As in $I$ let $\mathfrak{F}$ be the class of simple flexible power-associative partially stable algebras of degree two over an algebraically closed field of characteristic not 2,3 , or 5 . Let $A$ be an algebra in $₹$ and let 
$A^{+}$again denote the attached algebra with product $x \cdot y=1 / 2(x y+y x)$ and $[x, y]$ be the commutator product on $A . A^{+}$is a commutative algebra and is power-associative if $A$ is a power-associative algebra.

Tsai [16] has provied the following theorem which relates algebras in $\mathfrak{F}$ to their attached algebras:

Theorem 3. If $A$ is a flexible power-associative partially stable algebra of degree two over an algebraically closed field of characteristic not 2, 3, or 5, then $A$ is simple if and only if $A^{+}$is a simple algebra.

It is also known [1] that $A$ is $u$-stable if and only if $A^{+}$is $u$-stable. Also, $A$ is of degree two if and only if $A^{+}$has degree two. Hence if $A$ is in the class $\mathfrak{F}, A^{+}$is in the class $\mathbb{F}$ of commutative algebras described by Albert [4]. For the remaining part of this paper, assume that $A$ is in the class $\mathfrak{F}$.

Suppose $A$ is stable with respect to the idempotent $u$ and $A=A_{1}+A_{1 / 2}$ $+A_{0}$ is Peirce decomposition relative to $u$. Then $v=1-u$ is an idempotent orthogonal to $u$ and $A_{s}=A_{u}(s)=A_{v}(1-s)$ for $s=0,1,1 / 2$. It is known [14] that there exists an element $w$ in $A_{1 / 2}$ such that $w^{2}=1$. Let $B$ be the set of all elements $b$ of $C=A_{1}+A_{0}$ such that $b=(b \cdot w) \cdot w$. Then the following four lemmas are known concerning the role of $B$ in the structure of $A$.

Lemma 5 [14], [16]. $B^{+}$is a subalgebra of $C^{+}$, both $A_{0}{ }^{+}$and $A_{1}{ }^{+}$are isomorpbic to $B^{+}$, and $A_{1}=u B, A_{0}=v B, C^{+}=B^{+}+z \cdot B^{+}$where $z=u-v$. Furtbermore, $B^{+}=F_{p}\left[1, x_{1}, \cdots, x_{n}\right]$ is the truncated polynomial algebra on $n$ generators.

Let $y_{i}=y_{i} \cdot 1(i=0, \cdots, m)$ denote the elements of $A^{+}$used in Albert's description of the space $A_{1 / 2}$. Then we have

Lemma 6 [16]. The subspace $A_{1 / 2}$ can be represented as:

$$
A_{1 / 2}=\left(y_{0} \cdot B, \cdots, y_{m} \cdot B\right),
$$

where $A_{1 / 2}$ is the sum (not necessarily vector space direct sum) of $m+1$ bomomorphic images $y_{i} \cdot B$ of $B$.

The next result was first proved by Goldman [6] for $m=1$ and extended by Tsai [16] for arbitrary $m$.

Lemma 7. For any $b$ in $B$,

$$
y_{i} b=b y_{i}=y_{i} \cdot b \quad(i=0, \cdots, m) .
$$

Corollary [16]. For $a, b$ in $B$ and $i=0, \cdots, m$,

$$
a\left(y_{i} b\right)=\left(y_{i} b\right) a=a \cdot\left(y_{i} b\right) \text { and } a\left(y_{i} b\right)=a \cdot\left(y_{i} \cdot b\right)=y_{i} \cdot(a \cdot b) \text {. }
$$


In the next lemma we see how important a role $B$ plays in the structure of $A$.

Lemma 8 [16]. Every element in B commutes with every element in A and B is a commutative associative subalgebra of $A$.

By Lemma 8, $B=B^{+}$and hence Lemma 5 implies that $A_{0}=A^{+}, A_{1}=A_{1}{ }^{+}$ and $A_{0}=A_{1}$. Thus $\left(A_{0}+A_{1}\right)^{+}=A_{0}+A_{1}$ and again by Lemma $5, A_{0}+A_{1}=$ $B+z \cdot B=B+z B$ where $B+z B$ is a commutative associative algebra and $B$ is a truncated polynomial algebra. Now $A^{+}$is an algebra in the class $\mathbb{C}$ and hence the algebra $B$ is just the one used in the description for an algebra in $\Subset$. We shall freely use the results given in Lemmas $5-8^{\circ}$ without reference to the ir origin.

Lemma 9. If $a$ and $b$ are in $B$, then

$$
\left[y_{i} a, y_{j} b\right]=\left[y_{i}, y_{j}\right](a b) \quad(i, j=0, \cdots, m) .
$$

Proof. Letting $w=y_{i}, x=a$, and $y=y_{j} b$ in (13) we obtain $\left[y_{i} a, y_{j} b\right]=$ $y_{i} \cdot\left[a, y_{j} b\right]_{r}+a \cdot\left[y_{i}, y_{j} b\right]=\left[y_{i}, y_{j} b\right] a$. Applying the same procedure to $\left[y_{i} \cdot 1, y_{j} b\right]$ we see that $\left[y_{i} a, y_{j} b\right]=\left(\left[y_{i}, y_{j}\right] b\right) a=\left[y_{i}, y_{j}\right](a b)$. The last equality holds because $(x, a, b)=0$ for all $a, b$ in $B$ and $x$ in $A$. For if $x=c+d z+$ $\sum_{i=0}^{m} y_{i} b_{i}$ where $c, d$, and the $b_{i}$ 's are in $B$, then

$$
\begin{aligned}
(x a) b & =\left(c a+d a z+\sum_{i=0}^{m} y_{i} b_{i} a\right) b=c(a b)+(d z)(a b)+\sum_{i=0}^{m} y_{i} b_{i}(a b) \\
& =\left(c+d z+\sum_{i=0}^{m} y_{i} b_{i}\right)(a b)=x(a b)
\end{aligned}
$$

by the Corollary to Lemma 7 and the fact that $A_{0}+A_{1}$ is commutative and associative.

Lemma 9 reduces the study of the commutation properties of $A_{1 / 2}$ to the study of the $y_{i}$ 's under the commutator product. We can obtain a similar result for the case where the commutator has one element from $A_{1 / 2}$ and the other from $z B$.

Lemma 10. If $a$ and $b$ are in $B$ and $z=u-v$, then

$$
\left[y_{i} b, z a\right]=\left[y_{i}, z\right](a b) \quad(i=0, \cdots, m) .
$$

Proof. Let $w=y_{i}, x=b$, and $y=z a$ in (13). Then $\left[y_{i} b, z a\right]=y_{i} \cdot[b, z a]$ $+b \cdot\left[y_{i}, z a\right]=\left[y_{i}, z a\right] b$. Again using (13) with $w=z, x=b$, and $y=y_{i}$, $-\left[y_{i}, z a\right]=z \cdot\left[a, y_{i}\right]+a \cdot\left[z, y_{i}\right]=-\left[y_{i}, z\right] a$. Hence $\left[y_{i} b, z a\right]=\left(\left[y_{i}, z\right] a\right) b=$ $\left[y_{i}, z\right](a b)$. 
Next we prove an important result relating the multiplication in $A_{1 / 2}$ with multiplication between $A_{1 / 2}$ and $z B$.

Lemma 11. Let $a, b$, and $c$ be in $B$, then

$$
y_{i} a \cdot\left[y_{j} b, u c\right]=1 / 2(z c) \cdot\left[y_{i} a, y_{j} b\right] \quad(i, j=0, \cdots, m) .
$$

Proof. Let $x=y_{i} a, y=u c$, and $z=y_{j} b$ in (12) to obtain $\left[y_{i} a \cdot u c, y_{j} b\right]=$ $\left[y_{i} a, y_{j} b\right] \cdot(u c)+y_{i} a \cdot\left[u c, y_{j} b\right]$. Now $\left(y_{i} a\right) \cdot(u c)=\left(y_{i} a\right) \cdot 1 / 2(z c+c)=1 / 2\left(y_{i} a\right)_{c}$ and by Lemma 9 and the fact that $A_{1}+A_{0}$ is commutative and associative, $\left[\left(y_{i} a\right)_{c}, y_{j} b\right]=c\left[y_{i} a, y_{j} b\right]$. Hence $1 / 2 c\left[y_{i} a, y_{j} b\right]=u c \cdot\left[y_{i} a, y_{j} b\right]+y_{i} a \cdot\left[u c, y_{j} b\right]$ or equivalently

$$
y_{i} a \cdot\left[y_{j} b, u c\right]=\{(u-1 / 2) c\} \cdot\left[y_{i} a, y_{j} b\right] .
$$

But $u-1 / 2=1 / 2 z$ so $(14)$ is proved.

For the remainder of the paper let us assume that $y_{i} y_{j}$ is in $A_{0}+A_{1}$ for $i, j=0, \cdots, m$. For an algebra $A$ in the class $\mathfrak{f}$ satisfying this condition, the commutativity of $A$ is related to that of $A_{1 / 2}$ and to the multiplication between the idempotent $u$ and elements in $A_{1 / 2}$.

Theorem 4. The following are equivalent:

(i) $A$ is commutative.

(ii) $A_{1 / 2}$ is commutative.

(iii) $u a_{1 / 2}=a_{1 / 2} u$ for all $a_{1 / 2}$ in $A_{1 / 2}$.

Proof. If $A$ is commutative, then certainly $A_{1 / 2}$ is also commutative. Now assume that $A_{1 / 2}$ is commutative and suppose that $\left[y_{j}, u\right] \neq 0$ for some $j$. Let $x$ be any element in $A_{1 / 2}$, then $x=\sum_{i=0}^{m} y_{i} a_{i}$ where the $a_{i}$ 's are in $B$. By (14) with $b=c=1, x \cdot\left[y_{j}, u\right]=\left(\sum_{i=0}^{m} y_{i} a_{i}\right) \cdot\left[y_{j}, u\right]=\sum_{i=0}^{m} y_{i} a_{i} \cdot\left[y_{j^{\prime}} u\right]=$ $\sum_{i=0}^{m} 1 / 2 z \cdot\left[y_{i} a_{i}, y_{j}\right]=0$.

Therefore $x \cdot\left[y_{j}, u\right]=0$ for any $x$ in $A_{1 / 2}$. But $A^{+}$is simple and so has no nonzero $y$ in $A_{1 / 2}$ such that $x \cdot y=0$ for all $x$ in $A_{1 / 2}$. Hence $\left[y_{j}, u\right]=0$ for $j=0, \cdots, m$. Since $u=1 / 2(z+1)$, Lemma 10 implies that $\left[y_{i} a, u\right]=0$ for $a$ in $B$ and $i=0 ; \cdots, m$. Hence $u$ commutes with everything in $A_{1 / 2}$.

Now assume (iii). We already know that $A_{1}+A_{0}$ is a commutative subalgebra of $A$ and that the subalgebra $B$ of $A_{1}+A_{0}$ commutes with every element in $A$. Since $u$ commutes with everything in $A_{1 / 2}$, we know by Lemma 10 that $z B$ commutes with everything in $A$ and all that remains to be shown is that $A_{1 / 2}$ is commutative.

By (14) $1 / 2 z \cdot\left[y_{i}, y_{j}\right]=0(i, j=0, \ldots, m)$, and so $z\left(1 / 2 z\left[y_{i}, y_{j}\right]\right)=1 / 2\left[y_{i}, y_{j}\right]=$ 0 since $B+z B$ is commutative and associative and $z^{2}=1$. By Lemma 9 $\left[y_{i} a, y_{j} b\right]=\left[y_{i}, y_{j}\right](a b)=0$ and thus $A_{1 / 2}$ is commutative. Therefore $A$ is a commutative algebra. 
Lemma 12. $\left[y_{i} a, y_{j} b\right]$ is in $z B$ for $a, b$ in $B$ and $i, j=0, \cdots, m$.

Proof. Again by Lemma 9 it is sufficient to show the result for $\left[y_{i}, y_{j}\right]$. We know that $\left[y_{i}, y_{j}\right]$ is in $B+z B$. Now $\left[y_{j}, u\right]$ is in $A_{1 / 2}$ and hence $y_{i} \cdot\left[y_{j}, u\right]$ is in $B$ because of the way multiplication is defined in $A^{+}$. By equation (14) $z\left[y_{i}, y_{j}\right]$ is in $B$. Suppose $\left[y_{i}, y_{j}\right]=b_{1}+z b_{2}$ where $b_{1}$ and $b_{2}$ are in $B$. Then $z\left[y_{i}, y_{j}\right]=z\left(b_{1}+z b_{2}\right)=z b_{1}+b_{2}$ is in $B$. But then $z b_{1}$ must be in $B$ and thus $b_{1}=0$ since $z B \cap B=0$. Hence $\left[y_{i}, y_{j}\right]=z b_{2}$ is in $z B$.

The next two lemmas follow as consequences of Lemmas 11 and 12 .

Lemma 13. If $a, b$, and $c$ are in $B$, then $\left(y_{i} a\right) \cdot\left[y_{j} b, u c\right]=\left(y_{i} \cdot\left[y_{j}, u\right]\right)(a b c)$ $(i, j=0, \cdots, m)$.

Proof: By Lemma $9\left[y_{i} a, y_{j} b\right]=\left[y_{i}, y_{j}\right](a b)$ and by Lemma $12\left[y_{i}, y_{j}\right]=z d$ where $d$ is in $B$. Hence $\left[y_{i} a, y_{j} b\right]=(z d)(a b)=z(d a b)$. Then $z c\left[y_{i} a, y_{j} b\right]=$ $(z c)(z d a b)=b d a c=[z(z d)](a b c)=\left(z\left[y_{i}, y_{j}\right]\right) \cdot(a b c)$. Using (14), we have $\left(y_{i} a\right) \cdot\left[y_{j} b, u c\right]=1 / 2(z c)\left[y_{i} a, y_{j} b\right]=\left(1 / 2 z\left[y_{i}, y_{j}\right]\right)(a b c)$. But by (14) $1 / 2 z\left[y_{i}, y_{j}\right]=$ $y_{i} \cdot\left[y_{j}, u\right]$ so $\left(y_{i} a\right) \cdot\left[y_{j}, b, u c\right]=\left(y_{i} \cdot\left[y_{j}, u\right]\right)(a b c)$.

Lemma 14. Let $A_{1 / 2}=\left(y_{0} B, \cdots, y_{m} B\right), a$ and $b$ be in $B$ and $\left[y_{j}, u\right]=$ $\sum_{k=0}^{m} y_{k} a_{j k}(j=0, \cdots, m)$ where the $a_{j k}$ 's are in $B$. Then for $i, j=0, \cdots, m$,

$$
\sum_{k=0}^{m}\left[a D_{i k}\left(a_{j k} b\right)-b D_{i k}\left(a_{j k} a\right)\right]=0 .
$$

Proof. By Lemma $13\left(y_{i} a\right) \cdot\left[y_{j} b, u d\right]=\left(y_{i} \cdot\left[y_{j}, u\right]\right)(a b d)$ for all $a, b$ and $d$ in $B$ and $i, j=0, \cdots, m$. However, Lemma 10 allows us to write $\left[y_{j} b, u d\right]^{*}$ $=\left[y_{j}, u\right](b d)$. Hence

$$
\left(y_{i} a\right) \cdot\left\{\left[y_{j}, u\right](b d)\right\}=\left(y_{i} \cdot\left[y_{j}, u\right]\right)(a b d)
$$

or

$$
\left(y_{i} a\right) \cdot \sum_{k=0}^{m} y_{k}\left(a_{j k} b d\right)=\left(y_{i} \cdot \sum_{k=0}^{m} y_{k} a_{j k}\right)(a b d) .
$$

Using the multiplication defined for $A^{+}$we obtain

$$
\begin{aligned}
\sum_{k=0}^{m}\left[\left(y_{i} \cdot y_{k}\right)\left(a a_{i k} b d\right)\right. & \left.+a D_{i k}\left(a_{j k} b d\right)-\left(a_{j k} b d\right) D_{i k} a\right] \\
& =\sum_{k=0}^{m}\left[\left(y_{i} \cdot y_{k}\right)\left(a a_{j k} b d\right)-a_{j k} D_{i k}(a b d)\right] .
\end{aligned}
$$

Now each $D_{i k}$ is a derivation on $B$ so the above equation reduces to

$$
\sum_{k=0}^{m}\left[a D_{i k}\left(a_{j k} b d\right)-(b d) D_{i k}\left(a a_{j k}\right)\right]=0 \text {. }
$$


Letting $d=1$, we obtain (15). Our final result relates the commutativity of $A$ to properties of the derivations on $B$ used by Albert [4] in defining $A^{+}$.

Theorem 5. Suppose the subalgebra $B$ of $A$ bas $n$ generators $x_{1}, \cdots, x_{n}$ and $A_{1 / 2}=\left(y_{0} B, \cdots, y_{m} B\right)$. If for each $n=0, \cdots, m$ there exists some $i$ and some $b$ sucb that $x_{b} D_{i n}=s_{b n} \neq 0, s_{b n}$ in $F$, and $x_{b} D_{i t}=0$ for all $t \neq n$, then $A$ is commutative.

Proof. $\left[y_{j}, u\right]$ is in $A_{1 / 2}$ for $j=0, \cdots, m$. Suppose $\left[y_{j}, u\right]=\Sigma_{k=0}^{m} y_{k} a_{j k}$ $(j=0, \cdots, m)$ where the $a_{j k}$ 's are in $B$. By Lemma 14

$$
\sum_{k=0}^{m}\left[a D_{i k}\left(a_{j k} c\right)-c D_{i k}\left(a_{j k} a\right)\right]=0
$$

for all $a$ and $c$ in $B$. Let $j=q, n=r$ and let $i$ and $b$ be chosen as in the hypothesis of the theorem. Letting $c=1$ and $a=x_{b}$ we obtain

$$
\sum_{k=0}^{m}\left[x_{b} D_{i k} a_{j k}-1 D_{i k}\left(a_{j k} x_{b}\right)\right]=0 .
$$

Now $1 D_{i k}=0$ and $x_{b} D_{i k}=0$ except when $k=r$. So we have $s_{b r} a_{q r}=0$ and since $s_{b r} \neq 0, a_{q r}=0$. But $q$ and $r$ were arbitrary, so $a_{j k}=0(j, k=0, \cdots, m)$. Therefore $\left[y_{j}, u\right]=0$ for all $j=0, \cdots, m$ and by Theorem $4, A$ is commutative.

\section{REFERENCES}

1. A. A. Albert, Power-associative rings, Trans. Amer. Math Soc. 64 (1948), 552-593. MR 10, 349.

2. - A theory of power-associative commutative algebras, Trans. Amer. Math Soc. 69 (1950), 503-527. MR 12, 475.

3. - On commutative power-associative algebras of degree two, Trans. Amer. Math. Soc. 74 (1953), 323-343. MR 14, 614.

4. - On partially stable algebras, Trans. Amer. Math. Soc. 84 (1957), 430-443. MR 19, 1156.

5. - Addendum to the paper on partially stable algebras, Trans. Amer. Math. Soc. 87 (1958), 57-62. MR 19, 1157.

6. J. I. Goldman, A class of flexible power-associative algebras of degree two, M. S. Thesis, Illinois Institute of Technology, Chicago, Ill., 1963.

7. L. R. Harper, On differentiably simple algebras, Trans. Amer. Math. Soc. 100 (1961), 63-72. MR 24 \#A116.

8. E. Kleinfeld and L. A. Kokoris, Flexible algebras of degree one, Proc. Amer. Math. Soc. 13 (1962), 891-893. MR 25 \#5088.

9. L. A. Kokoris, Power-associative commutative algebras of degree two, Proc. Nat. Acad. Sci. U. S. A. 38 (1952), 534-537. MR 14, 11.

10. —, On nilstable algebras, Proc. Amer. Math. Soc. 9 (1958), 697-701.

MR 20 \#3902.

11. - Nodal non-commutative Jordan algebras, Canad. J. Math. 12 (1960), 488-492. MR 22 \#6834.

12. - Flexible nilstable algebras, Proc. Amer. Math. Soc. 13 (1962), 335-340. MR 25 \#1190. 
13. R. H. Oehmke, On flexible algebras, Ann. of Math. (2) 68 (1958), 221-230. MR $21 . \# 5664$.

14. - On flexible power-associative algebras of degree two, Proc. Amer. Math. Soc. 12 (1961), 151-158. MR 25 \#103.

15. - Flexible power-associative algebras of degree one, Proc. Nat. Acad. Sci. U. S. A. 63 (1969), 40-41. MR $42 \# 1870$.

16. C. E. Tsai, Flexible partially stable algebras, Trans. Amer. Math. Soc. 122 (1966), 48-63. MR 33 \#4113.

DEPARTMENT OF MATHEMATICS, ILLINOIS INSTITUTE OF TECHNOLOGY, CHICAGO, ILLINOIS 60616 60626

Current address: Department of Mathematics, Loyola University, Chicago, Illinois 